\title{
Optical Filters to Exclude Non-Doppler-Shifted Light in Fast Velocimetry
}

\author{
D. Goosman, F. Avara, J. Wade, A. Rivera
}

This article was submitted to $25^{\text {th }}$ International Congress on High Speed Photography and Photonics, Beaune, France, September 29 - October 4, 2002

\section{August 22, 2002}

U.S. Department of Energy

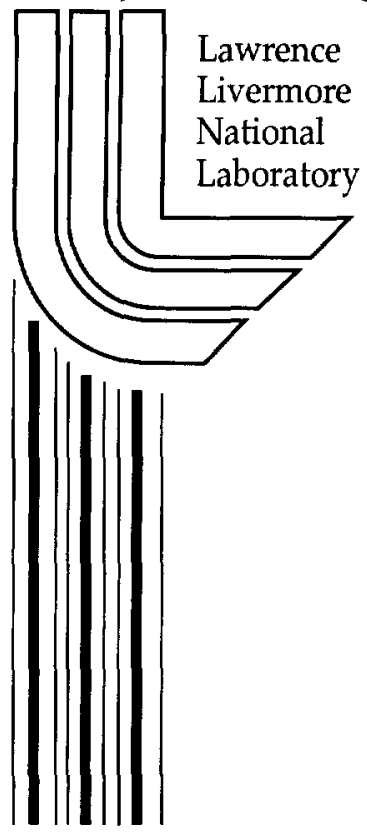




\section{DISCLAIMER}

This document was prepared as an account of work sponsored by an agency of the United States Government. Neither the United States Government nor the University of California nor any of their employees, makes any warranty, express or implied, or assumes any legal liability or responsibility for the accuracy, completeness, or usefulness of any information, apparatus, product, or process disclosed, or represents that its use would not infringe privately owned rights. Reference herein to any specific commercial product, process, or service by trade name, trademark, manufacturer, or otherwise, does not necessarily constitute or imply its endorsement, recommendation, or favoring by the United States Government or the University of California. The views and opinions of authors expressed herein do not necessarily state or reflect those of the United States Government or the University of California, and shall not be used for advertising or product endorsement purposes.

This is a preprint of a paper intended for publication in a journal or proceedings. Since changes may be made before publication, this preprint is made available with the understanding that it will not be cited or reproduced without the permission of the author.

This work was performed under the auspices of the United States Department of Energy by the University of California, Lawrence Livermore National Laboratory under contract No. W-7405-Eng-48.

This report has been reproduced directly from the best available copy.

Available electronically at http://www.doc.gov/bridge

Available for a processing fee to U.S. Department of Energy

And its contractors in paper from

U.S. Department of Energy

Office of Scientific and Technical Information

P.O. Box 62

Oak Ridge, TN 37831-0062

Telephone: (865) 576-8401

Facsimile: (865) 576-5728

E-mail: reports@adonis.osti.gov

Available for the sale to the public from

U.S. Department of Commerce

National Technical Information Service

5285 Port Royal Road

Springfield, VA 22161

Telephone: (800) 553-6847

Facsimile: (703) 605-6900

E-mail: orders@ntis.fedworld.gov

Online ordering: http://www.ntis.gov/ordering.htm

\section{OR}

Lawrence Livermore National Laboratory

Technical Information Department's Digital Library

http://www.llnl.gov/tid/Library.html 


\title{
Optical filters to exclude non-Doppler-shifted light in fast velocimetry-REV-1
}

\author{
David Goosman, George Avara, James Wade and Anthony Rivera \\ The authors are with Lawrence Livermore National Laboratory \\ P.O. Box 808, L-281, Livermore, CA 94550 \\ 925-422-1630, fax 925-422-2382
}

\begin{abstract}
We frequently measure velocity-time histories of dynamic experiments. In some, the Doppler-shifted light is often weak compared to non-shifted light reflected from stationary surfaces and imperfections in components. With our Fabry-Perot (FP) based systems which handle multiple frequencies, data is lost where the fringes coincide; if we had used an intensity-measuring VISAR system, it would probably fail. We designed a facility for doing experiments under such conditions by selectively eliminating most of the non-shifted light. Our first filter excluded non-shifted light by a factor of 300 when manually tuned, and by 150 when run in an auto-tuning mode. It used a single 50 mm diameter FP as the filter with a spacing of $1.65 \mathrm{~mm}$ and reflectivities of $77 \%$, and filters five channels prior to use in one of our 5-beam velocimeters. One use of the filter system was to embed optical fibers in long sections of explosives to make continuous detonation velocity-time histories. We have carried out many such tests with this filter, and two without. A special single-beam filter was constructed with a $40 \%$ efficiency for shifted light that rejected non-shifted light by 4 million times, with a bandpass of a few $\mathrm{GHz}$.
\end{abstract}

Keywords: velocimetry, filters, Doppler, Fabry-Perot

\section{INTRODUCTION}

We have used both VISAR and Fabry-Perot velocimeter systems at LLNL to measure the time dependent velocity of surfaces accelerated by gas guns, exploding foils and explosives ${ }^{1}$. Since the FP systems can handle more than one frequency of return light, we have used only those systems for the last 17 years. Our manybeam system was described at a recent Congress ${ }^{2}$, at which time we had 10 of the 20 channels of recorders in use.

Several years ago, the first author was shown records taken at LANL by W. Hemsing ${ }^{3}$ done to measure continuous detonation velocities within explosives, by monitoring the frequency of light reflected from the moving crushed fiber end within the explosive. Two experiments were then carried out at LLNL in 1998. The data made sense, but was frequently obscured by non-Doppler shifted light that was an order of magnitude stronger than the data sought.

We devised a filtration system to mostly eliminate the non-shifted light to obtain better records. Placing fibers in explosives to measure continuous detonation velocities may give large amounts of undesired non-Doppler shifted light scattering from fiber imperfections and connections. We designed and constructed a special filtration system, based upon angled fiber connections and a Fabry-Perot etalon that reduces this undesired light by a factor of more than 100 .

\section{EMBEDDED FIBER OPTICS}

A typical embedded fiber experiment with a fiber being enveloped by a detonation wave is shown in fig. 1 . 


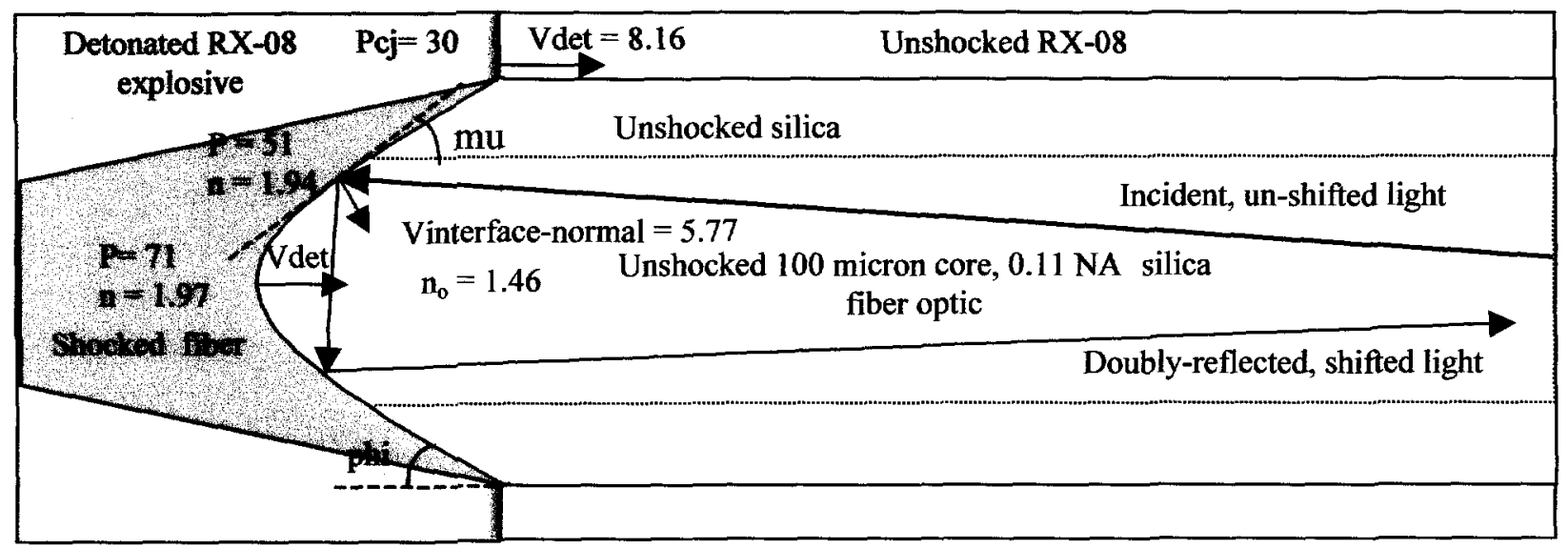

Figure 1. Approximate shape of the end of a crushed fiber embedded in RX-08HD explosive. Pressures are in GPa, as calculated by Souers ${ }^{4}$, the refractive index is $\mathrm{N}$, and velocities are in $\mathrm{mm} / \mathrm{usec}$.

It is a challenge for hydrocodes to have the resolution to model a 100-micron diameter fiber end, and to include

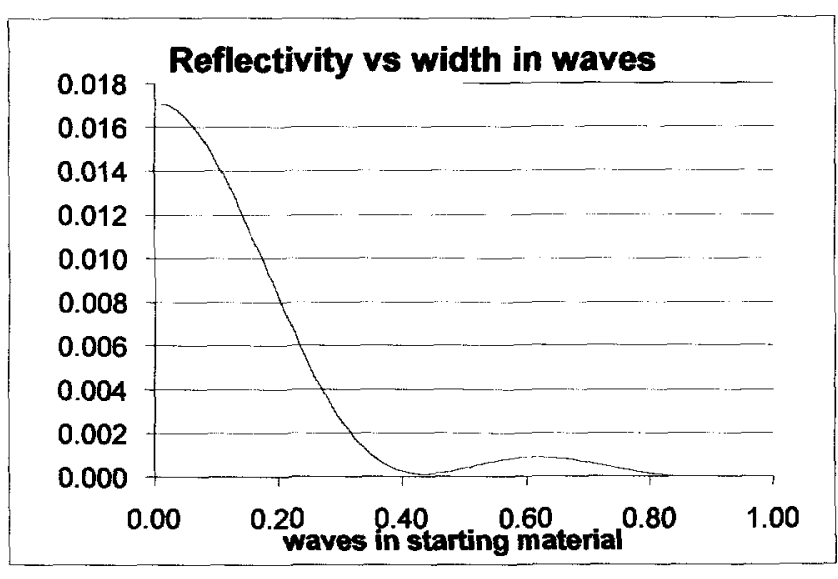
enough explosive volume for accuracy. If the shock speed in the silica fiber core does not outrun the detonation velocity, $V_{\text {det }}$, a self-similar cup-shaped interface propagates to the right at the speed $=\mathrm{V}_{\mathrm{det}}$. For this example, the index rises from 1.46 to about 1.9 at the interface. The first author has calculated how much the reflectivity drops if the index change is not rapid with distance, by numerically solving the wave equations for propagation through a material of varying index. If the interface transition region is thicker than about $20 \%$ of the wavelength in the unshocked material, then the reflection from this interface is less than half of that for a sharp interface, as shown in figure 2 . The uncertainty in the transition width is one of the two reasons why it is difficult to predict the amount of reflected light.

Figure 2. The calculated reflectivity of a boundary whose index rises linearly with distance from 1.46 to 1.9 , as a function of the width of the transition region, measured in units of waves in the starting material.

The other reason is the uncertainty in the shape of the interface. If the center is nearly flat, then some light within the 0.11 NA can reflect once from this flatter portion and still be within the $0.11 \mathrm{NA}$ of the fiber. This contribution is estimated to be about le-5 of the incident light. A more important contribution is the doubly reflected light, which samples an annular region of the interface. The area of this useful annulus is usually much larger than the area of the flat part mentioned earlier, but the two reflections mean much less intensity per unit area than from a single reflection. A Monte-Carlo program showed that for interfaces with a region that has a near 45 degree slope, most of the returned light is from two reflections. Granulation of the interface, if it occurs, could reduce the reflected light that is within 0.11 NA.

The frequency of light Doppler shifting from a solid surface, such as a diffuse metal, depends only upon the particle velocity, incident laser and collection direction vectors, and not upon the surface normal direction, as shown experimentally ${ }^{(5)}$. However, a shocked interface in a transparent material also has a Doppler-shifted reflection, which is known by normal-incidence experiments done at LLNL to be a direct measure of the shock speed, and not the particle velocity behind the shock front. But we care about the correct shift for light incident at an angle to the shock front. We assume that in this case also it is only the interface velocity and not the particle velocity that matters. Then the fractional frequency shift (FFS) from each reflection at the interface would be given by the vector product: 


$$
\mathrm{FFS}=(\text { Index of unshocked silica }) / \mathbf{c}^{*} \mathbf{V}_{\text {interface }}{ }^{*}\left(\mathbf{K}_{\text {reflected }}-\mathbf{K}_{\text {incident }}\right),
$$

where bold fonts indicates vectors, $\mathrm{c}$ is the speed of light, and the $\mathrm{K}$ vectors are of unit magnitude and simply indicate direction. The index of refraction of the unshocked silica multiplies the normal velocity vector product, because the interface moving at $V_{\text {interface }}$ is overtaking wavecrests in the unshocked silica which are closer together than they are in air by a factor of $n=1.46$. $V_{\text {interface }}=V_{\text {det }}$ if the solution is self-similar as mentioned above.

Many hydrocalculations for self-similar solutions indicate that the particle velocity behind the shock in the silica is very nearly normal to the interface. In this case it is easy to show that

$$
V_{\text {interface-normal }}=V_{\text {det }} * \sin (m u) \text {, }
$$

where $\mathrm{mu}$ is the angle of the interface relative to the fiber axis.

Single shock data in silica give the relation between shock speed and pressure, which is the $U_{\mathrm{s}}-\mathrm{P}$ curve. The crushed-fiber calculations done by C. Soures ${ }^{4}$ of LLNL, which are not single shock experiments, indicate that the pressure at the axis on the interface is close to the value on the $U_{s}-P$ curve, if one sets $U_{S}=V_{\text {det }}$. For example, at the point shown on figure $1,71 \mathrm{GPa}$ is close to the pressure on the silica $U_{\mathrm{s}}-\mathrm{P}$ curve for a $U_{\mathrm{s}}$ value of $8.16 \mathrm{~mm} / \mathrm{usec}$.

The apparent velocity varies from $n_{0}{ }^{*} V_{d e t}$ to a smaller value depending upon the $\mathrm{k}$ directions. The Monte-Carlo program mentioned above also tallies the total Doppler shift from each interface reflection and gives a spectrum of shifts for the light that is reflected and transported back through the fiber to the velocimeter analysis table. For 0.11 NA fibers, the result is that the average shift due to the finite NA is quite small: the apparent velocity should be multiplied by 1.0014 to correct for the NA effect.

Doubling the NA of the fiber will increase the Doppler broadening of the reflected line by a factor of about 4 as well as collecting more light. But our Fabry system cannot use more light than that carried by a 100 micron 0.11 NA fiber anyway ${ }^{2}$. However, 0.22 NA fibers may in fact make more useful light than 0.11 fibers, and unless the shape of the interface is known, one needs to answer this question experimentally.

\section{THE NON-SHIFTED LIGHT FILTRATION SYSTEM}

Non-shifted light can arise from imperfections within the fiber, reflections from the fiber-air interfaces and scattering

from dust on the optical elements. To greatly reduce the fiber-air interface reflections, we angled the entrance ends of the fibers carrying high laser power to the explosive. A nine-degree angle on the end of the fiber was sufficient to assure that reflected light missed the optical lens illuminating that fiber. The same angling was used for the one connector between the fiber embedded into the explosive and the fiber going to the filter. This scheme reduces the connector reflections from about $4 \%$ for normal connectors to about 100 times less.

To further reduce non-Doppler-shifted light, a filtration system based upon a closely-spaced Fabry Perot interferometer was designed. A Fabry interferometer can have its spacing set to transmit the non-shifted light and reflect the rest. Denoting the intensity reflectivities of mirrors 1 and 2 as $R_{1}$ and $R_{2}$, the intensity transmission as $T_{i}$, and the absorption coefficient of the mirror as A, then it is straightforward to show that for non-striped interferometers, the following relationships are valid:

$$
T_{i}+R_{i}+A=1 \quad(i=1,2) \text { for each mirror. }
$$

For light incident at an angle theta to the normal to the mirrors, the general expressions for the transmission and reflection of the interferometer, for identical mirrors and reflectivities above about $60 \%$, are ${ }^{6}$

$$
\text { Transmission }=\mathrm{T}^{2} /\left((1-\mathrm{R})^{2}+4 \mathrm{R} \sin ^{2}(\mathrm{phi} / 2)\right), \quad \text { and }
$$


where

$$
\text { Reflection }=R+T\left(T R-2 R \cos (p h i)+2 R^{2}\right) /\left((1-R)^{2}+4 R \sin ^{2}(p h i / 2)\right),
$$

$$
\mathrm{phi}=4(\mathrm{pi}) \mathrm{H}(\mathrm{nu}) \cos (\text { theta }) / \mathrm{c}
$$

is the phase change due a round-trip pass through the interferometer, and

$$
\mathbf{R}=\mathbf{R}_{1}=\mathbf{R}_{2}
$$

is the reflectivity of each mirror. $\mathrm{H}$ is the mirror spacing, nu and $\mathrm{c}$ are the frequency and speed of the light and theta is the polar angle between the light and the surface normal to the FP mirrors.

The beam should be nearly normal to the mirrors to achieve maximum transmission. In our 5-beam system, which uses one FP, we keep all of the fibers close together with the same polar angle with respect to the normal to the FP mirrors to minimize filtration inefficiencies due to angular spread. The maximum and minimum transmissions are

$$
\begin{aligned}
& T_{\max }=T^{2} /(1-R)^{2}, \text { and } \\
& T_{\min }=T^{2} /(1+R)^{2}
\end{aligned}
$$

Useful expressions for the reflected intensity when the transmission is peaked are:

$$
\text { Reflection (at Tmax) }=\mathrm{R}+\mathrm{T}\left(\mathrm{TR}-2 \mathrm{R}+2 \mathrm{R}^{2}\right) /(1-\mathrm{R})^{2} \text {, }
$$

and the reflected intensity when the transmission is near minimum, which is

$$
\text { Reflection (at } T \min )=R+T\left(T R+2 R+2 R^{2}\right) /(1+R)^{2}
$$

The idea is to use the reflected beam from the filter interferometer which will have a much reduced non-shifted component and send it to the usual velocimeter analyser table (with its own interferometer) for analysis. Table 1 illustrates numerical examples.

$\begin{array}{llclllll} & & \text { A } & 0.00200 & 0.00200 & 0.00200 & 0.00200 & 0.00000 \\ & & \text { R1 } & 0.71000 & 0.77000 & 0.85000 & 0.90000 & 0.90000 \\ & & \text { R2 } & 0.71000 & 0.77000 & 0.85000 & 0.90000 & 0.90000 \\ & & \text { T1 } & 0.28800 & 0.22800 & 0.14800 & 0.09800 & 0.10000 \\ \text { Unshifted } & \text { At peak transmission } & \text { Transm } & 0.28800 & 0.22800 & 0.14800 & 0.09800 & 0.10000 \\ \text { Unshifted } & \text { At peak transmission } & \text { Refl } & 0.98625 & 0.98268 & 0.97351 & 0.96040 & 1.00000 \\ \text { Shifted } & \text { At minimum transm } & \text { Refl } & 0.00003 & 0.00006 & 0.00015 & 0.00036 & 0.00000 \\ \text { Shifted } & \text { At minimum transm } & \text { Transm } & 0.02837 & 0.01659 & 0.00640 & 0.00266 & 0.00277\end{array}$

Table 1. Numerical examples of the transmission and reflection formulae.

This table shows shifted and non-shifted amounts for 4 combinations of mirror reflectivities ranging from 0.71 to 0.90 , assuming perfectly flat mirrors. The last column shows that if the absorption $\mathrm{A}$ is zero, which is unrealistic, the reflectance at peak transmission falls to zero.

The critical row is the reflection of non-shifted light when the filter is set at peak transmission, which varies from 0.00003 to 0.00036 . We want this value to be low so the experiment is not contaminated with too much non-shifted light. Reducing the mirror reflectivities from 0.9 to 0.71 helps in two ways. First of all, the non-shifted reflectance gets 12 times smaller, and it becomes less sensitive to having exactly the correct mirror spacing, $H$. The slight penalty for this is that the reflectance of the shifted light, which we want to be large, drops from 0.995 to 0.969 .

A plot of the reflectance and transmission of a filter with $R_{1}=R_{2}=0.71$ is shown at the bottom of figure 3 as a function of frequency. The latter of which has been converted to the product of the interface velocity and the index of refraction, as detailed in eqn. (1). 


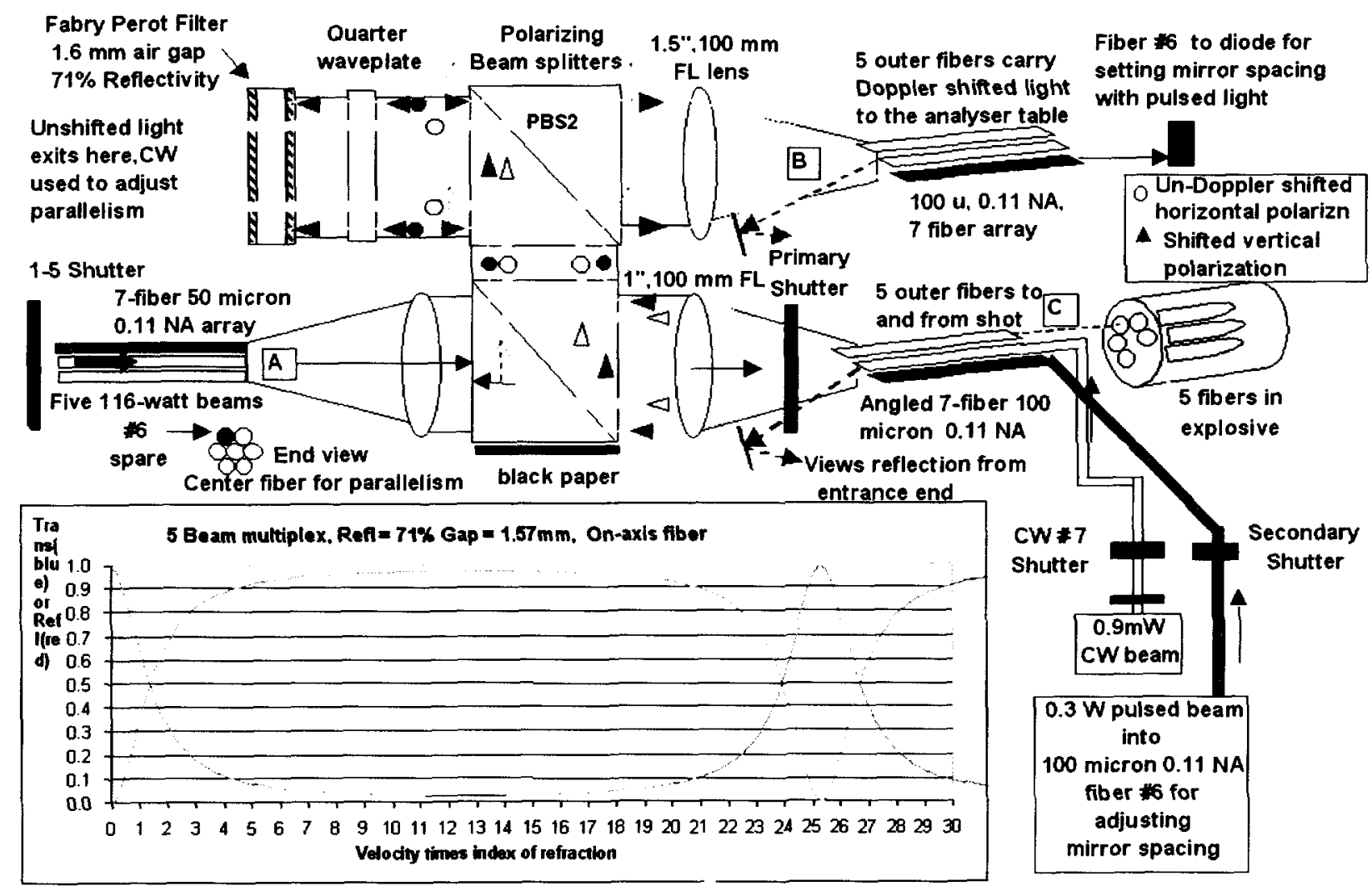

Figure 3. The system to reduce non-shifted light. The upper and lower curves for $V$ near 0 are transmission and reflection curves.

The graph in figure 3 assumes that the filter mirrors were first made parallel by using $\mathrm{CW}$ light into fiber \# 7 . Then the mirror spacing was set to minimize the non-shifted reflection for pulsed light injected into the fiber \#6. This is because the exact wavelengths of the $\mathrm{CW}$ and pulsed laser are different and because the shot fibers and fiber \#6 are off axis, whereas fiber $\# 7$ is on axis. Off-axis fibers have minimum reflection at mirror spacings about $0.005^{*}$ waves larger than for on-axis fibers.

\section{OPERATION OF THE FILTER SYSTEM}

Light from the laser is injected into 5 of the 7 fibers in holder A, as shown in figure 3 . We have repeatedly put 116 watts of $532 \mathrm{~nm}$ light for 50 microseconds into each one without damage. This light is unpolarized by virtue of having traversed several meters of step-index 50-micron diameter and 0.11 numerical aperture fiber. The light is collimated as well as can be done (limited by the fiber diameter) by a $100-\mathrm{mm}$ focal length $25 \mathrm{~mm}$ diameter achromat lens. Half of this light passes through the lower polarizing beamsplitter cube. The rest is wasted into a piece of black paper (but could be used by a more elaborate 10 -fiber version of this system). The $50 \%$ passing through the cube is imaged onto a similar 7 -fiber chuck $\mathrm{C}$, made of 100 micron cores, instead of 50 , to facilitate high power alignment. Then there is less chance of a poorly-aligned beam burning the polyimide on chuck $\mathrm{C}$, which is angled to prevent reflections. In addition, this angling aids a viewing system, which uses a CCD camera with a tiny mirror that is placed to intercept this reflected beam (but little of the incident beam). The viewer allows us to see the 7 fiber cores and the 5 beam spots simultaneously to assure proper registration. All other connectors are angled.

Light from each fiber reflects from the moving interface in the explosive, is Doppler-shifted and returns along the same fiber to chuck $\mathrm{C}$. The horizontally polarized half of that light is reflected by the lower cube to the upper one. There it is $100 \%$ reflected to a quarter-wave plate and into the filter. The spacing of the filter has been set to efficiently transmit the non-shifted light (from connector reflections, bubbles and imperfections in the fiber) and reflect efficiently the 
shifted light from the shock in the explosive. This shifted light passes through the quarter-wave plate a second time, giving it a vertical polarization allowing it to pass through the upper cube and be focussed onto the 7-fiber chuck B, which is also angled. A CCD views reflected light, allowing alignment of the chuck B. The shifted light entering the fibers of chuck B goes to the usual manybeam velocimeter analyser table ${ }^{2}$ to record the data. One unit of light fed to the analyser table on these 0.11 NA fibers is equivalent to about 2.5 units of light fed to the same table on the usual $0.22 \mathrm{NA}$ fibers. This is because the standard nested lens probes used on most shots collect light into $0.22 \mathrm{NA}$ fibers and the analyser table normally uses only the 0.11 NA part of that light. Presently the normal analyser table input is a 1-meter long 0.22 NA 100 micron fiber. We have found that these short fibers, when injected with 0.11 NA light, emit at about 0.13 NA. We are building a 0.11 NA replacement fiber chuck for this 1 meter section.

\section{FINE-TUNING THE FILTER AND MAXIMIZING THE EXTINCTION RATIO}

With only the $\mathrm{CW}$ beam on, we inject into the center fiber of chuck $\mathrm{C}$. With low reflectivity mirrors, tuning for parallelism on a reflected beam is more sensitive than with the transmitted beam. One of us, (GA), noticed in the transmitted pattern both the usual bright beam and a weaker beam below it that looked like the reflected beam. The latter is conjugate to the transmitted one, when the one is bright, the conjugate is dark. We transported that weak image of the reflected beam into another CCD viewer so that it could be seen at both the filter and control rooms. The extinction is the ratio of reflected intensity at maximum vs. minimum as we adjust the common spacing between the filter mirrors. This can be measured at the control room by via a power meter connected to fiber \#7 of chuck B. When tuning locally, we obtained a ratio of 300 . We found that if the filter is tuned initially locally, we could then go to the control room and keep it in tune sufficiently to obtain a ratio of 200 . We used the tuning code written by one of us (GA), and used a very fine range on the piezo stack voltage driver.

The exact frequency of the pulsed laser is different than that of the $\mathrm{CW}$ laser used to make the mirrors parallel. Thus we need the pulsed beam to set the filter spacing to resonantly transmit the non-shifted pulsed light. Peronnel are not in the filter room during a shot, so we monitored the reflected pulsed light from fiber \#6 of chuck B at the control room, and could adjust the spacing up to the last few seconds prior to the detonation of the shot.

\section{EXPERIMENTS WITH AND WITHOUT THE FILTER}

Figure 4 is an early result with a fiber in detasheet explosive, prior to use of the filter. The detonation velocity lines are visible but heavily obscured by intense non-shifted light. Even the SBS lines (downshifted by $32.8 \mathrm{GHz}$ due to high power) are equal in strength to the shifted lines.

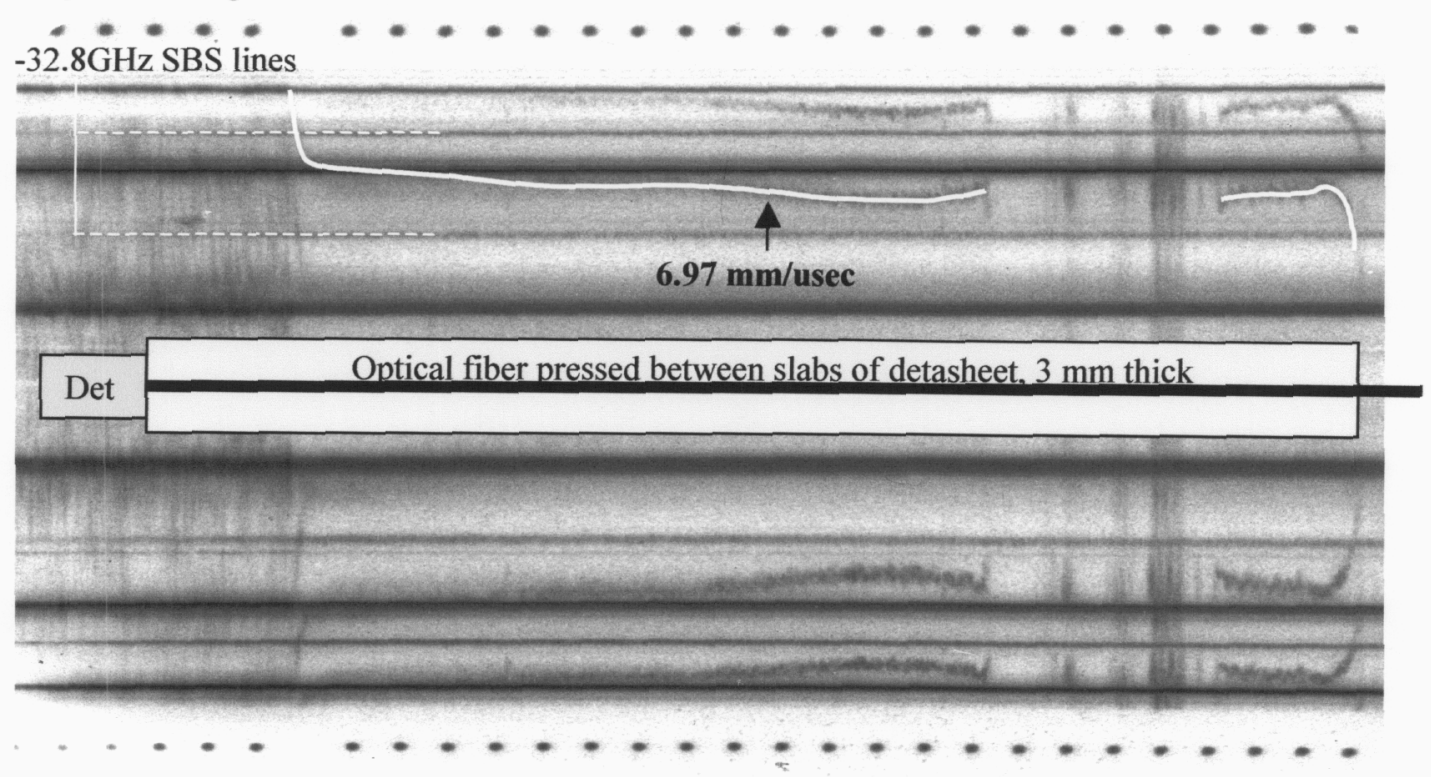

Figure 4. A Fabry-Perot velocimetry record taken without the filter. Undesired non-shifted light dominates. 
Figure 5 shows the data taken with LX-17 explosive. The baseline non-shifted light at the beginning is mostly due to reflection from the explosive near the end of the embedded fiber. After the shock arrives, this source disappears, and the remaining non-shifted light (shown for about $300 \mathrm{nsec}$ in figure 5) is intentionally produced light, since a small amount is useful for calibration. We first use the filter to make the non-shifted light negligible, and then inject downstream of the filter a small but controlled amount of non-shifted light from the laser.

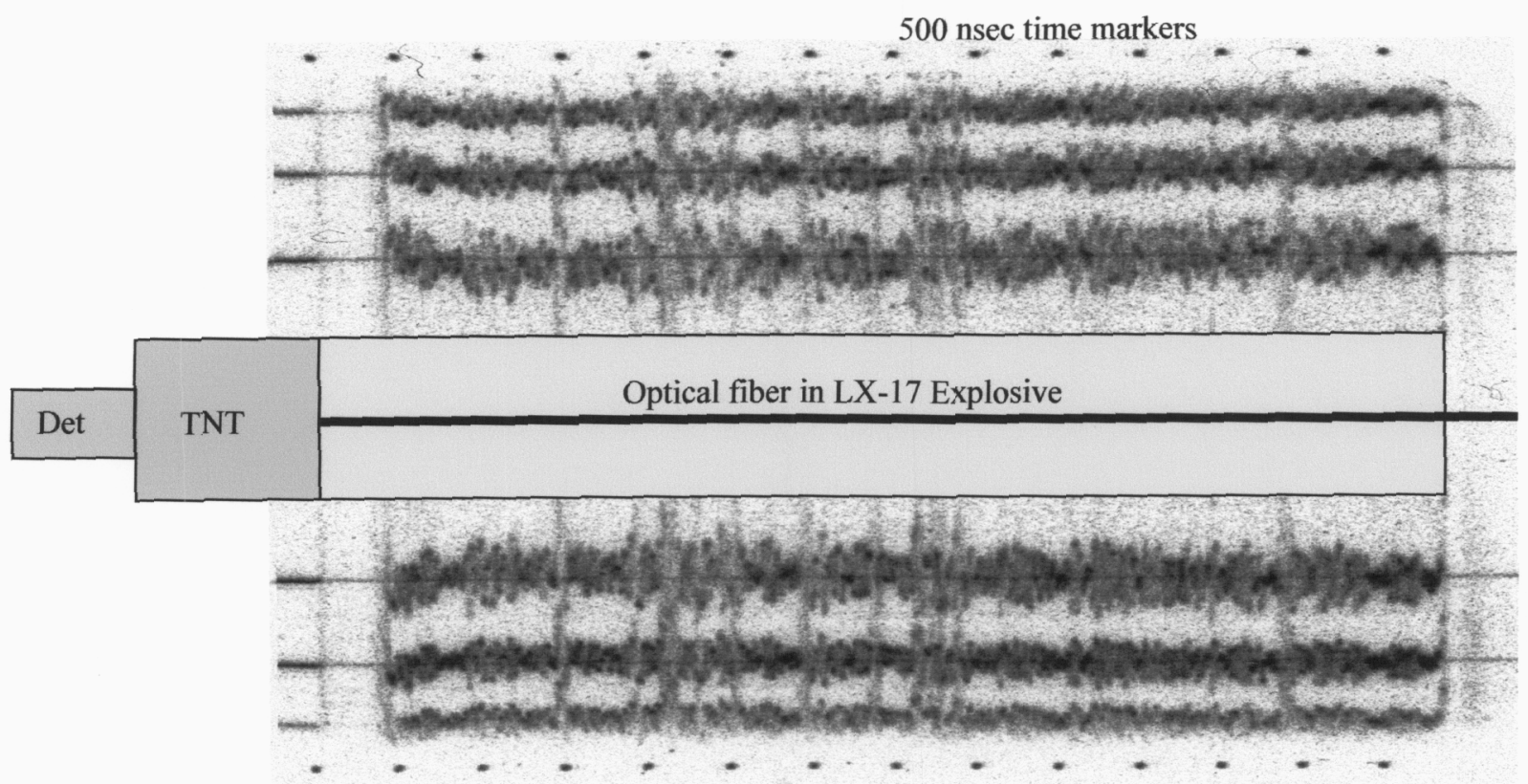

Figure 5. A record taken on LX-17 explosive with the filter. The detonation velocity just exceeds 12 orders of interference. Undesired non-shifted light has been eliminated.

A filter such as this could be used in conjuction with an intensity-measuring VISAR velocimeter to eliminate the nonshifted light, which can cause analysis errors if large enough and unforseen ${ }^{7}$.

\section{HIGH EXTINCTION RATIO FILTER}

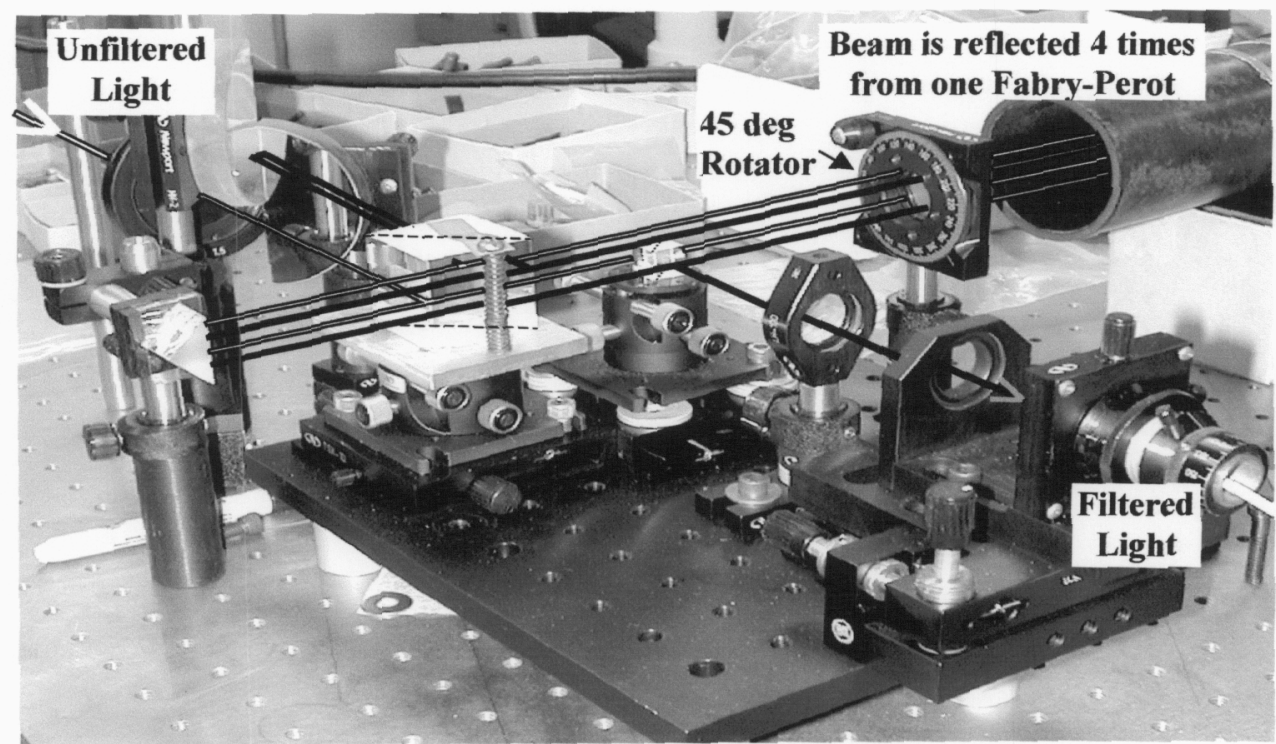

Figure 6 shows a filter we designed for an experiment suggested by J. Osher of LLNL, searching for an extremely weak shifted component in the presence of intense unshifted light. Measurements showed that the filter reflects $40 \%$ of the desired shifted light, and rejects unshifted light by a factor exceeding 4 million, with a bandpass of a few $\mathrm{GHz}$.

Figure 6. The high extinction ratio filter. 


\section{HIGH EFFICIENCY FILTER}

Figure 7 shows another version of the filter we are constructing, using both polarizations of the return light, in order to roughly double the efficiency. With special fibers, one could in principle triple the efficiency.

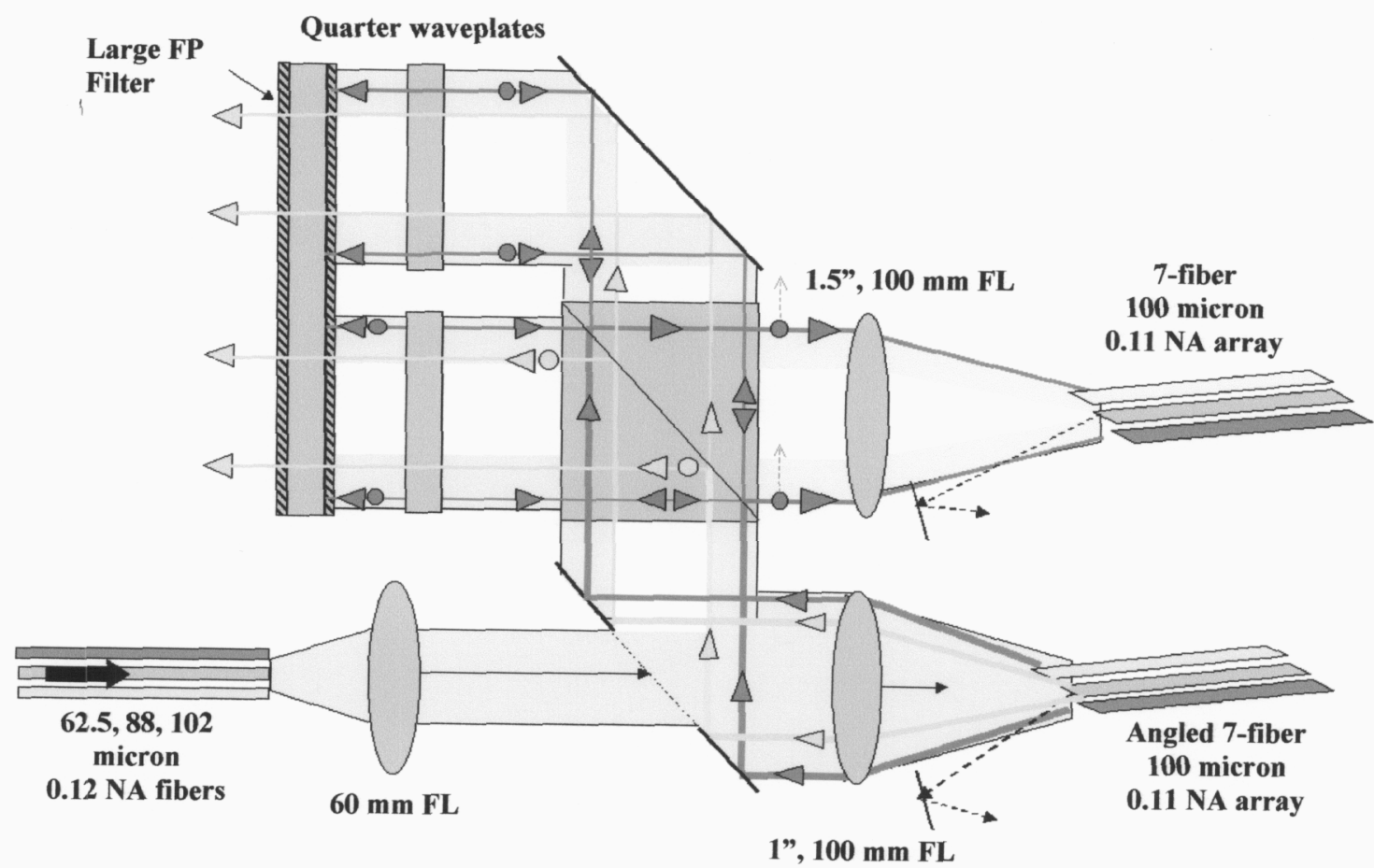

Figure 7. The proposed higher-efficiency filter. Doppler-shifted light is shown by the darker, wider lines.

\section{REFERENCES}

1. D. R. Goosman, "Formulas for Fabry-Perot velocimeter performance using both stripe and multifrequency techniques", Appl. Opt. 30 (27), 3907-3923 (1991).

2. D. R. Goosman, G. Avara, L. Steinmetz, C. Lai and S. Perry, "Manybeam velocimeter for fast surfaces", SPIE proceedings on the $22^{\text {nd }}$ Int. Congress on High Speed Photography and Photonics, 2869, 1070-1079 (1996).

3. Willard Hemsing, private communication

4. C. Souers, private communication

5. D. R. Goosman, A. M. Frank, H. H. Chau, and N. L. Parker, "Fabry-Perot Velocimetry Techniques: Is Doppler Shift Affected by Surface Normal Direction?", Proc. High Speed Photography, Videography, and Photonics", Soc. Of Photo-Optical Instr. Engrs. 427, 127 (1983).

6. J. M. Vaughan, "The Fabry-Perot Interferometer" (Adam Hilger,Bristol, 1989), Chap. 3.

7. D. R. Goosman, George R. Avara and Stephen J. Perry, "Efficient optical probes for fast surface velocimetry: multiple frequency issues for Fabry and VISAR methods", SPIE proceedings on the $24^{\text {th }}$ Int. Congress on High Speed Photography and Photonics, 4183, 413-423 (2000).

Work performed under the auspices of the U.S. Department of Energy by Lawrence Livermore National Laboratory under Contract W-7405-Eng-48. 